Proc. 15th Int. Conference on Defects Recognition, Imaging and Physics in Semiconductors, Warsaw, Poland 2013

\title{
Kelvin Force Microscopy Characterization of Corona Charged Dielectric Surfaces
}

\author{
D. MARINSKiY ${ }^{a}$, P. EDElman ${ }^{a}$ AND A.D. SNIDER ${ }^{b}$ \\ ${ }^{a}$ Semilab SDI, 10770N. 46th St., Ste E700, Tampa, FL, USA \\ ${ }^{b}$ University of South Florida, 4202 East Fowler Avenue, Tampa FL, USA
}

\begin{abstract}
Ionic diffusion of $\left(\mathrm{H}_{2} \mathrm{O}\right)_{n}^{+}$and $\mathrm{CO}_{3}^{-}$on $\mathrm{SiO}_{2}$ surfaces has been quantified using Kelvin force microscopy measurement of ion distribution change after small spot corona charge. For both positive and negative ionic species, the concentration profiles versus time follow the two-dimensional surface diffusion enabling a determination of corresponding diffusion coefficients. On a thermally grown $\mathrm{SiO}_{2}$ surface, diffusion coefficients of $\left(\mathrm{H}_{2} \mathrm{O}\right)_{n}^{+}$and $\mathrm{CO}_{3}^{-}$ ions were $2.2 \times 10^{-11} \mathrm{~cm}^{2} / \mathrm{s}$ and $4.8 \times 10^{-12} \mathrm{~cm}^{2} / \mathrm{s}$, respectively. On a chemically cleaned $\mathrm{SiO}_{2}$ surface, diffusion coefficients of $\left(\mathrm{H}_{2} \mathrm{O}\right)_{n}^{+}$and $\mathrm{CO}_{3}^{-}$ions were $7.5 \times 10^{-9} \mathrm{~cm}^{2} / \mathrm{s}$ and $2.4 \times 10^{-9} \mathrm{~cm}^{2} / \mathrm{s}$, respectively. Mathematical analysis of the surface potential decay yields an additional parameter - capacitance equivalent thickness.
\end{abstract}

DOI: 10.12693/APhysPolA.125.997

PACS: 77.55.-g, 73.61.-r, 68.37.Ps, 73.25.+i

\section{Introduction}

The corona-Kelvin based technique has been used as a non-contact, preparation free replacement of MOS and Schottky barrier capacitance-voltage (CV) measurements. It allows quantification of dielectric and interface properties such as dielectric capacitance and electrical thickness [1-4], density of interface states [2-4], and leakage across the dielectric [2-6]. Traditionally, a large spot corona-Kelvin approach is applied to unpatterned monitor wafers with blanket dielectric layers $[2,4,5]$. A miniaturization of both the Kelvin probe and corona deposition area has been introduced that extended the corona-Kelvin metrology to small spot measurements on product (i.e. patterned) silicon IC wafers and specifically to scribe-line test sites, which are $100 \mu \mathrm{m} \times 100 \mu \mathrm{m}$ or smaller $[4,7]$. Modified Kelvin force microscopy (KFM) with a probe of about $10 \mu \mathrm{m}$ is used for surface potential measurements while the corona deposition diameter is reduced below $100 \mu \mathrm{m}$ by employing a small aperture corona gun with electrostatic ion focusing [7].

In large spot corona-Kelvin measurements the surface potential is stable after corona charge deposition providing that the leakage current across dielectric is small enough not to neutralize the corona charge. As the corona deposition area is reduced below $100 \mu \mathrm{m}$, the surface potential on some dielectrics begins to exhibit a characteristic time decay (Fig. 1) that is related to a different phenomenon. Our work demonstrates that the origin of this potential decay is the lateral diffusion of corona ions away from the center of deposition. We analyze the rate of potential decay as a two-dimensional diffusion mechanism.

In this paper we present results of ionic diffusion on two types of $\mathrm{SiO}_{2}$ surfaces, i.e. the surface after thermal oxidation and the surface after chemical cleaning. The experimental surface potential distribution profiles are analyzed using fundamental and modified solutions to the diffusion equation. A modified solution accounts

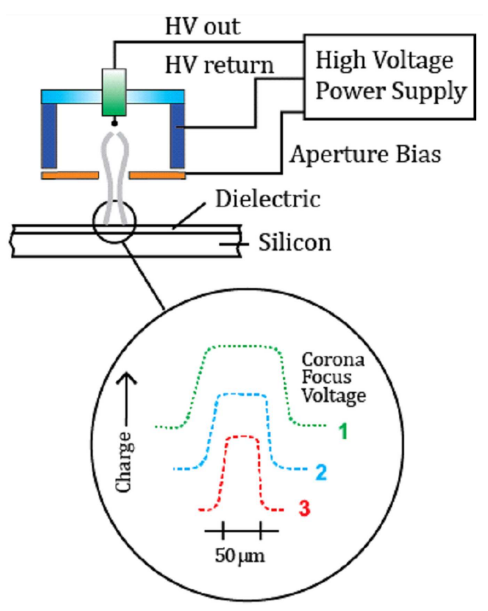

Fig. 1. Schematic drawing of apparatus used for deposition of corona charge on a dielectric surface. Increasing corona bias voltage $1<2<3$ decreases the diameter of corona spot.

for a non $\delta$-function initial corona distribution. Lastly, an exact mathematical solution is presented. We demonstrate that the analysis of the surface potential decay yields two important parameters: capacitance equivalent thickness of the dielectric, CET, and diffusion coefficient of corona ions on the dielectric surface.

\section{Experimental}

\subsection{Sample preparation}

$P$-type silicon wafers with a nominal $100 \AA$ thermal $\mathrm{SiO}_{2}$ layer were used in this study. Wafer resistivity was $8-12 \Omega \mathrm{cm}$. One wafer was measured without additional surface treatment. The other was treated in $\mathrm{SC} 1$ solution (Standard Clean 1, $\mathrm{NH}_{4} \mathrm{OH}: \mathrm{H}_{2} \mathrm{O}_{2}: \mathrm{H}_{2} \mathrm{O}=1: 1: 5$ at $80^{\circ} \mathrm{C}$ for $10 \mathrm{~min}$ ) followed by a DI water rinse. $\mathrm{SC} 1$ cleaning is commonly used in silicon IC processing and is known to produce an $-\mathrm{OH}$ terminated $\mathrm{SiO}_{2}$ surface $[8,9]$. 


\subsection{Measurement apparatus}

\subsubsection{Deposition of corona ions}

Corona ions are generated by a corona discharge in air under atmospheric pressure. The corona apparatus consists of a point electrode in a charge confining cylinder. A high potential of 3 to $10 \mathrm{kV}$ is applied to the point electrode. A wafer is placed on a grounded chuck and a $500 \mu \mathrm{m}$ aperture is placed between the electrode and the wafer to control the area of corona charge deposition. Corona apparatus is schematically shown in Fig. 1. Positive ions, $\left(\mathrm{H}_{2} \mathrm{O}\right)_{n}^{+}$, are generated under positive bias conditions, and negative ions, $\mathrm{CO}_{3}^{-}$, are generated under negative bias conditions $[10,11]$.

A corona charge diameter of $\approx 80 \mu \mathrm{m}$ was achieved using electrostatic focusing, i.e. by application of ion repelling bias voltage to the aperture, see the inset in Fig. 1 . Aperture bias voltage was $\approx 300 \mathrm{~V}$. Surface ion density was controlled by controlling high voltage applied to the point electrode and the charging time. Initial ion density was in the range $0.4-0.6 \mu \mathrm{C} / \mathrm{cm}^{2}$. For an $\mathrm{SiO}_{2}$ dielectric this corresponds to an electric field of $1.2-1.6 \mathrm{MV} / \mathrm{cm}$, i.e. much lower than the tunneling field range for charge leakage across $\mathrm{SiO}_{2}$ dielectric $[4,5,12]$ that could neutralize the corona charge.

It has been reported [13-16] that surface conductivity of $\mathrm{SiO}_{2}$ is a strong function of humidity. One can expect that ionic diffusion on the surface also depends on humidity. Therefore for an accurate comparison of the two $\mathrm{SiO}_{2}$ surface conditions experiments were performed in a clean room with controlled temperature and humidity at $40 \% \mathrm{RH}$.

\subsubsection{Measurements of surface potential}

Kelvin force microscopy (KFM) is used for surface potential measurements. A probe of $10 \mu \mathrm{m}$ diameter enables measurements of surface potential distribution with high spatial resolution. The probe operates at a resonance frequency range above $100 \mathrm{kHz}$, enabling fast response time. Measurement accuracy below $0.5 \mathrm{mV}$ is achieved at a probe to surface distance of $1 \mu \mathrm{m}$. KFM measurements were done on a FAaST 300 SL system [17].

The surface potential is measured before and after deposition of the corona charge. The change in surface potential is related to the surface density of ions as

$$
q N(r, t)=C \Delta V_{\mathrm{CPD}}(r, t),
$$

where $q$ is the elementary charge, $r$ is the distance from the center of the corona deposition area, $N(r, t)$ is the concentration of ions on dielectric surface $\left[\mathrm{cm}^{-2}\right]$, $C$ is the capacitance per unit surface area $\left[\mathrm{F} \mathrm{cm}^{-2}\right]$, and $\Delta V_{\mathrm{CPD}}(r, t)$ is the change in the surface potential with respect to the value before corona charging [V]. For an oxide/semiconductor interface, the total capacitance includes capacitance of the dielectric layer $C_{\text {diel }}$ and the capacitance of semiconductor space charge layer $C_{\mathrm{SC}}$ [18]: $C^{-1}=C_{\text {diel }}^{-1}+C_{\mathrm{SC}}^{-1}$. Under surface accumulation or inversion conditions $C_{\mathrm{SC}} \gg C_{\text {diel }}$ and therefore the capacitance is approximately equal to the dielectric capacitance, $C_{\text {diel }}$. Capacitance of the space charge layer is also increased for highly doped wafers and by illuminating the sample with high intensity light [2]. For example, capacitance of a $100 \AA \mathrm{SiO}_{2}$ dielectric is $\approx 0.35 \mu \mathrm{F} / \mathrm{cm}^{2}$ and the conditions $C_{\mathrm{SC}} \gg C_{\text {diel }}$ is satisfied when silicon surface potential is $<-0.2 \mathrm{~V}$ (deep accumulation) or $>0.8 \mathrm{~V}$ (strong inversion). Therefore measuring under strong illumination conditions is important to minimize contribution of $C_{\mathrm{SC}}$ to the total capacitance. In the analysis that follows, we approximate $C \approx C_{\text {diel }}$.

\section{Theory and calculation}

The ion density $N(r, \theta, z, t)$ spreads according to the diffusion equation $\frac{\partial N}{\partial t}=\nabla \cdot(\stackrel{\leftrightarrow}{D} \cdot \nabla N)$. The vertical component of diffusion is negligible and the phenomenon is modeled as radially symmetric. The governing equation becomes

$$
\frac{\partial N}{\partial t}=D \nabla^{2} N(r, t)=D\left(\frac{\partial^{2} N}{\partial r^{2}}+\frac{1}{r} \frac{\partial N}{\partial r}\right) .
$$

The solution depends on initial conditions, i.e. initial ion distribution and dose. We will discuss three cases below.

\subsection{Fundamental solution}

For an initial $\delta$-function distribution the solution has a Gaussian form

$$
N(r, t)=\frac{N_{0}}{4 \pi D t} \exp \left(\frac{-r^{2}}{4 D t}\right),
$$

where $N_{0}$ is the initial dose of corona ions, $r$ is the radius from the center of ion deposition, $t$ is the time after ion deposition, and $D$ is the surface diffusion coefficient.

The change of the surface potential distribution is described by

$$
\Delta V_{\mathrm{CPD}}(r, t)=\frac{q N_{0}}{C_{\mathrm{diel}} 4 \pi D t} \exp \left(\frac{-r^{2}}{4 D t}\right) .
$$

Fitting the experimental surface potential distribution to Eq. (4) enables extraction of the diffusion coefficient, $D$. Moreover, knowledge of the dielectric capacitance, $C_{\text {diel }}$, and initial ion dose, $N_{0}$, is not required for diffusion coefficient calculation if only the exponential term in Eq. (4) is considered.

\subsection{Modified Gaussian distribution}

A modified solution is proposed to account for a non $\delta$-function initial distribution. If the initial distribution can be approximated by a Gaussian distribution, then a modified solution is

$$
\Delta V_{\mathrm{CPD}}(r, t)=\frac{q N_{0}}{C_{\mathrm{diel}} 4 \pi D(t+\tau)} \exp \left(\frac{-r^{2}}{4 D(t+\tau)}\right) .
$$

Justification for the modified solution is as follows: at time $t=\tau$ the $\delta$-function will look like the initial surface potential distribution. So the solution $\Delta V_{\mathrm{CPD}}(r, t)$ can be approximated as a fundamental solution (Eq. (4)) starting $\tau$ seconds earlier. This is shown schematically in Fig. 2. Parameter $\tau$ can be determined from the time dependence of the pre-exponential term in Eq. (5).

\subsection{Exact solution to $2 D$ diffusion equation}

Deposition of corona ions results in a uniform distribution over a circle of radius $R=40 \mu \mathrm{m}$. The wafer diameter is $300 \mathrm{~mm}$, i.e. much larger than the diameter 

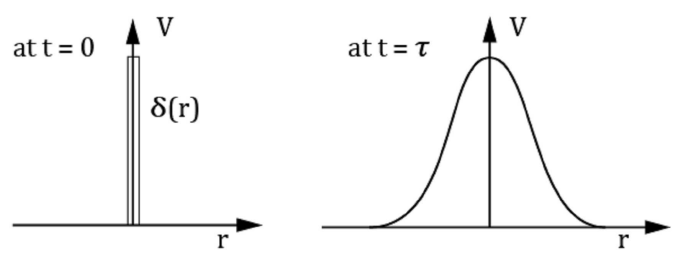

Fig. 2. Corona ion distribution at time $t=0$ and at time $t=\tau$. After $\tau$ seconds the initial $\delta$-distribution changes to Gaussian.

of the corona ion deposition area. It can be taken as infinitely remote for the duration of diffusion. The initial ion density produces the initial surface charge density $Q_{0}=q \times N_{0} . R$ and $N_{0}$ are determined by the charging conditions.

The solution to the diffusion Eq. (2) satisfying the initial condition is given by USFKAD [19]:

$$
N(r, t)=N_{0} \int_{0}^{\infty} A(k) J_{0}(k r) \mathrm{e}^{-k^{2} D t} \mathrm{~d} k,
$$

where

$$
A(k)=\int_{0}^{R} J_{0}(k r) k r \mathrm{~d} r=R J_{1}(k R) .
$$

The voltage dependence at $r=0$ can be described as (Appendix A):

$$
\Delta V(t)=\frac{h}{\varepsilon \varepsilon_{0}} q N_{0}-\frac{h}{\varepsilon \varepsilon_{0}} q\left(N_{0}-N_{\infty}\right) \mathrm{e}^{-R^{2} / 4 D t},
$$

where $N_{\infty}=N(0, \infty)$ is the ion density at time $t=\infty$, $h$ is the thickness of the dielectric layer, $R$ is the radius of initial corona charge deposition circle.

From Eq. (7) it follows that the plot of $t \times \ln \left(-t^{2} \dot{V}(t)\right)$ versus $t$, defined as a Snider plot, should be a straight line (Appendix A):

$$
\begin{aligned}
& t \ln \left(-t^{2} \dot{V}(t)\right)=\left(\ln \frac{q\left(N_{0}-N_{\infty}\right) R^{2} h}{4 \varepsilon \varepsilon_{0} D}\right) t-\frac{R^{2}}{4 D} \\
& \quad=\text { slope } \cdot t+\text { intercept. }
\end{aligned}
$$

The slope and intercept can be estimated by linear regression, enabling the extraction of $D$ and $h$ :

$$
\begin{aligned}
D & =\frac{-R^{2}}{4 \times \text { intercept }}, \\
h & =\left(\Delta V(t)+\frac{4 D}{R^{2}} \mathrm{e}^{\text {slope }} \mathrm{e}^{\text {intercept } / t}\right) \frac{\varepsilon \varepsilon_{0}}{q N_{0}} .
\end{aligned}
$$

\section{Results and discussion}

Time evolution of the surface potential distribution after charging with positive $\left(\mathrm{H}_{2} \mathrm{O}\right)_{n}^{+}$and negative $\mathrm{CO}_{3}^{-}$ions are shown in Figs. 3, 4.

\subsection{Diffusion coefficient from the fundamental and modified solutions}

Individual voltage-distance profiles are fitted to the fundamental (Eq. (4)) and the modified (Eq. (5)) solutions. Parameter $\tau$ for the modified solution is obtained from the voltage transient at $r=0$, i.e. $V(0, t)$. Figure 5 shows fitting of $\Delta V(0, t)$ to $1 /(t+\tau)$ for SC1 cleaned and thermal $\mathrm{SiO}_{2}$ surfaces. Table I summarizes $\tau$ values

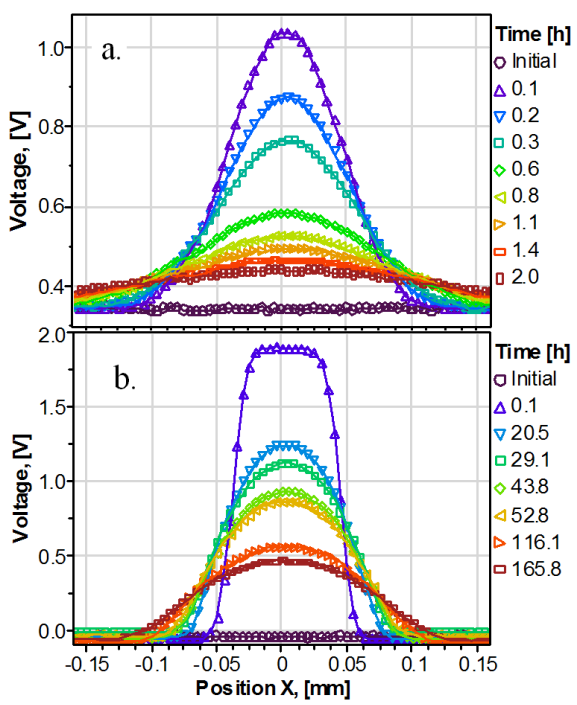

Fig. 3. Kelvin force microscopy profiles of the surface potential distribution for different times after deposition of positive corona ions, $\left(\mathrm{H}_{2} \mathrm{O}\right)_{n}^{+}$, on $\mathrm{SC} 1$ cleaned (a) and thermal (b) $\mathrm{SiO}_{2}$ surfaces.

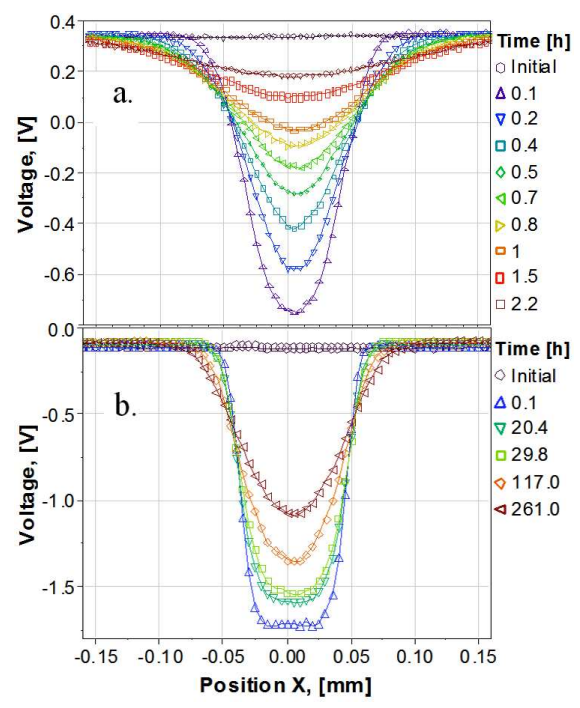

Fig. 4. KFM profile of the surface potential distribution versus position $X$ for different times after deposition of negative corona ions, $\mathrm{CO}_{3}^{-}$, on $\mathrm{SC} 1$ cleaned (a) and thermal (b) $\mathrm{SiO}_{2}$ surfaces.

obtained for each surface and corona ion condition. Diffusion coefficients calculated for each voltage distribution profile are shown in Figs. 6 and 7.

Diffusion coefficients calculated from the fundamental solution show a characteristic time dependence. This is due to the simplified assumption of the initial $\delta$-function corona ion distribution. It can be seen (Figs. 6,7 ) that for longer times the calculated diffusion coefficient saturates and approaches values obtained from the modified solution. This is expected since for longer times $t \gg \tau$ and therefore Eqs. (4) and (5) should give a similar dif- 


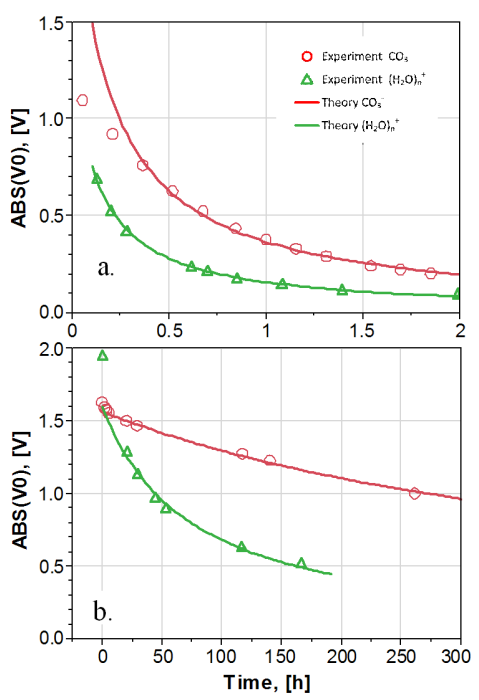

Fig. 5. Voltage transient at $r=0$ for SC1 cleaned (a) and thermal (b) $\mathrm{SiO}_{2}$ surfaces. Fitting to $1 /(t+\tau)$ enables extraction of the parameter $\tau$.

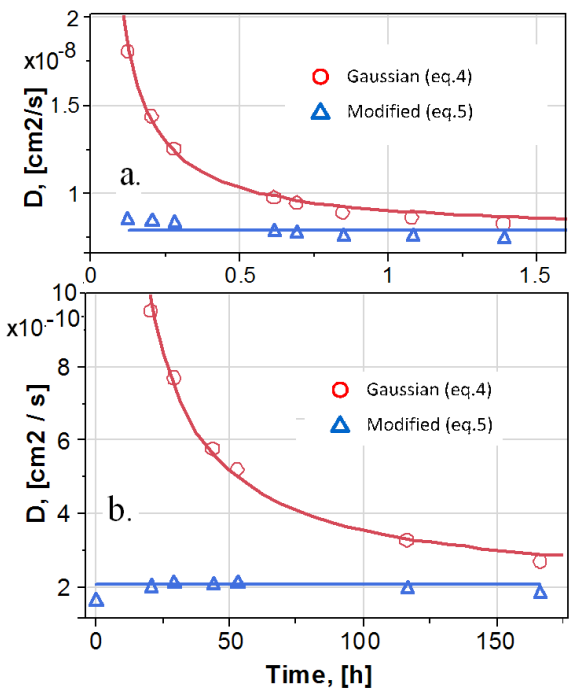

Fig. 6. Diffusion coefficient for each voltage distribution profile shown in Fig. 3 for positive corona ions, $\left(\mathrm{H}_{2} \mathrm{O}\right)_{n}^{+}$, on $\mathrm{SC} 1$ cleaned (a) and thermal (b) $\mathrm{SiO}_{2}$ surfaces.

fusion coefficient. We note that for the $\mathrm{SC} 1$ cleaned $\mathrm{SiO}_{2}$ surface parameter $\tau$ is less than $0.2 \mathrm{~h}$ (Table I) while the change of surface potential distribution is monitored for $2 \mathrm{~h}$ (Figs. 3a, 4a). The condition $t \gg \tau$ is satisfied and indeed diffusion coefficients obtained from the fundamental and modified solutions show approximately the same values.

On the thermal $\mathrm{SiO}_{2}$ surface, parameter $\tau$ is $74 \mathrm{~h}$ and $482 \mathrm{~h}$ for diffusion of positive and negative corona ions respectively, while the change of surface potential distribution is monitored for up to $300 \mathrm{~h}$. The longest measurement time is less than $2 \times \tau$ and diffusion coefficient obtained from the fundamental and modified solutions

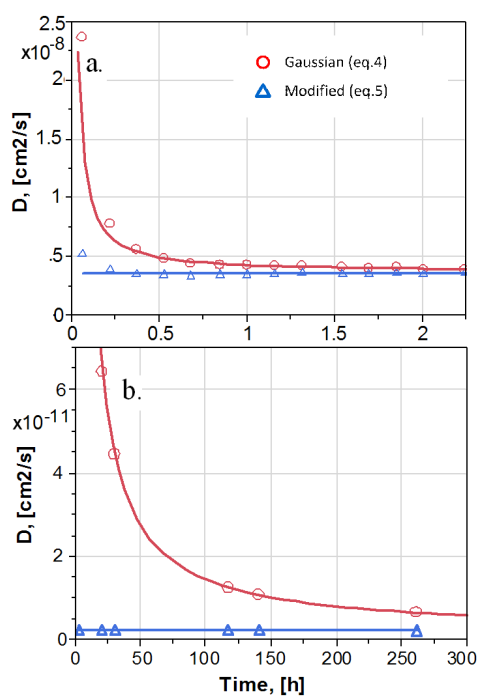

Fig. 7. Diffusion coefficient for each voltage distribution profile shown in Fig. 4 for negative corona ions, $\mathrm{CO}_{3}^{-}$, on $\mathrm{SC} 1$ cleaned (a) and thermal (b) $\mathrm{SiO}_{2}$ surfaces.

TABLE I

Parameter $\tau$ obtained from the fitting of $V(0, t)$ to $1 /(t+\tau)$.

\begin{tabular}{c|c|c}
\hline \hline Ion & Surface & $\tau[\mathrm{h}]$ \\
\hline \multirow{2}{*}{$\left(\mathrm{H}_{2} \mathrm{O}\right)_{n}^{+}$} & SC1 & 0.14 \\
& thermal & 74.12 \\
\hline \multirow{2}{*}{$\mathrm{CO}_{3}^{-}$} & SC1 & 0.19 \\
& thermal & 482.1
\end{tabular}

still shows a difference. For positive corona ions the difference is $\approx 1.5$ times while for the negative corona ions the difference is $\approx 3$ times. Nevertheless, from the fundamental solution the diffusion coefficient can be obtained by extrapolation to time $t=\infty$.

Diffusion coefficients obtained from the fundamental and modified solutions are summarized in Table II. Ionic diffusion on the $\mathrm{SC} 1$ treated $\mathrm{SiO}_{2}$ surface is much faster than that on $\mathrm{SiO}_{2}$ after thermal processing. We observed more than 2 orders of magnitude increase in the diffusion coefficient on the $\mathrm{SC} 1$ treated surface.

TABLE II

Diffusion coefficient $\left[\mathrm{cm}^{2} / \mathrm{s}\right]$ obtained from the fundamental and modified solutions.

\begin{tabular}{c|c|c|c}
\hline \hline Ion & Surface & $\begin{array}{c}\text { Fundamental } \\
(\text { Eq. (4)) }\end{array}$ & Modified (Eq. (5)) \\
\hline \multirow{2}{*}{$\left.\mathrm{H}_{2} \mathrm{O}\right)_{n}^{+}$} & $\mathrm{SC} 1$ & $(8.2 \pm 0.1) \times 10^{-9}$ & $(7.8 \pm 0.2) \times 10^{-9}$ \\
& thermal & $(2.8 \pm 0.2) \times 10^{-11}$ & $(2.1 \pm 0.1) \times 10^{-11}$ \\
\hline $\mathrm{CO}_{3}^{-}$ & $\begin{array}{c}\mathrm{SC} 1 \\
\text { thermal }\end{array}$ & $\begin{array}{c}(4.2 \pm 0.1) \times 10^{-9} \\
(9.8 \pm 0.1) \times 10^{-12}\end{array}$ & $(2.6 \pm 0.8) \times 10^{-9}$ \\
& \multicolumn{2}{|c|}{$(2.5 \pm 0.1) \times 10^{-12}$}
\end{tabular}

The diffusion coefficient for $\left(\mathrm{H}_{2} \mathrm{O}\right)_{n}^{+}$is higher than that for $\mathrm{CO}_{3}^{-}$. The higher diffusion coefficient for $\left(\mathrm{H}_{2} \mathrm{O}\right)_{n}^{+}$is consistent with its smaller atomic weight. 
Lastly, the simplifying assumption of the initial $\delta$-function distribution is justified by the ease of the experimental data analysis and from the fact that for longer times ions spread over a distance much larger than the initial corona distribution, and therefore the initial corona distribution can be well approximated by the $\delta$-function.

\subsection{Diffusion coefficient and CET \\ from the exact solution}

Solution of diffusion Eq. (2) using USFKAD predicts that the plot of $t \times \ln \left(-t^{2} \dot{V}(t)\right)$ versus $t$, defined as a Snider plot, should be a straight line. Data for SC1 cleaned and thermal $\mathrm{SiO}_{2}$ surfaces are shown in Fig. 8 . Let us note the excellent linearity of the data. $R^{2}$ of the linear fit is $>0.997$ for all cases (Table III). The diffusion coefficient and CET are calculated using the intercept and slope of the linear fit (Eqs. (9), (10)) and the results are summarized in Table III.
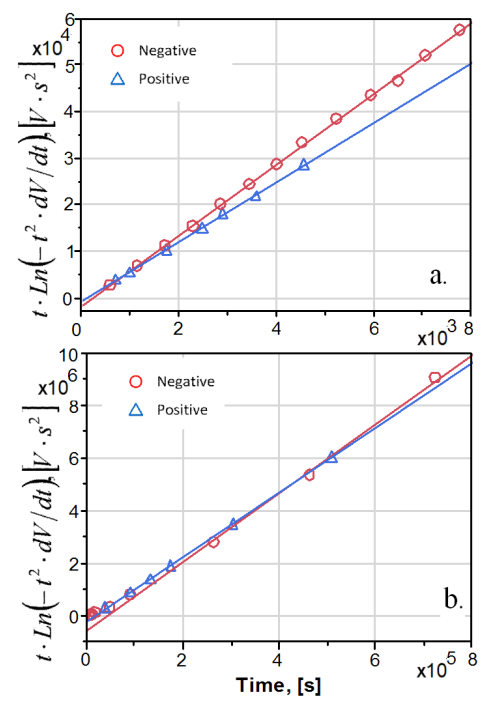

Fig. 8. Snider plot for (a) SC1 cleaned and (b) thermal $\mathrm{SiO}_{2}$ surfaces.

Diffusion coefficient and CET obtained from TABLE $\mathrm{F}$ IULt mathematical solution.

\begin{tabular}{c|c|c|c|c}
\hline \hline Ion & Surface & $D\left[\mathrm{~cm}^{2} / \mathrm{s}\right]($ Eq. $(9))$ & $R^{2}$ & CET $[\AA]$ \\
\hline \multirow{2}{*}{$\left(\mathrm{H}_{2} \mathrm{O}\right)_{n}^{+}$} & SC1 & $(7.5 \pm 0.5) \times 10^{-9}$ & 0.9995 & 94.7 \\
& thermal & $(2.2 \pm 0.3) \times 10^{-11}$ & 0.9999 & 97.7 \\
\hline \multirow{2}{*}{$\mathrm{CO}_{3}^{-}$} & SC1 & $(2.4 \pm 0.2) \times 10^{-9}$ & 0.9994 & 92.1 \\
& thermal & $(4.8 \pm 0.9) \times 10^{-12}$ & 0.9979 & 95.4
\end{tabular}

Initial conditions include the radius of the corona deposition area, $R$, and the initial ion density, $N_{0}$. From Eqs. (9) and (10) it follows that the calculation of diffusion coefficient only depends on the radius $R$, while the calculation of CET only depends on $N_{0}$. This simplifies the experimental setup: if one is interested in a diffusion coefficient, then only the radius of the initial corona deposition area must be known; if one is interested in a CET parameter, then only the initial corona dose must be known/calibrated.
CET calculated for the thermal and $\mathrm{SC} 1$ cleaned $\mathrm{SiO}_{2}$ surface shows good agreement with the nominal dielectric thickness of $100 \AA$. The small difference observed for positive and negative bias conditions is attributed to the contribution of the silicon space charge layer, while the difference between the $\mathrm{SC} 1$ cleaned and thermal $\mathrm{SiO}_{2}$ surfaces is attributed to slight etching of $\mathrm{SiO}_{2}$ by $\mathrm{SC} 1$ solution [20, 21].

The diffusion coefficient obtained from the solution of the $2 \mathrm{D}$ diffusion equation shows good agreement with the data obtained from the analysis of voltage distribution profiles using the fundamental and modified solutions.

\section{Conclusions}

We have presented a method to quantify ion surface diffusion coefficients on dielectric surfaces. The ions are deposited onto the dielectric surface by small spot corona charging and the charge profile is measured as a function of time by the Kelvin force microscopy measurement of the surface voltage.

We found that for both positive, $\left(\mathrm{H}_{2} \mathrm{O}\right)_{n}^{+}$, and negative, $\mathrm{CO}_{3}^{-}$, ionic species the concentration profile versus time follows two-dimensional surface diffusion enabling calculation of the corresponding surface diffusion coefficients. We have analyzed experimental data using three approaches, which were different by assumption of initial ion distribution, namely, fundamental, modified, and exact solutions. Diffusion coefficients calculated using each approach show good agreement with each other. These coefficients are different for as grown oxide and chemically cleaned $\mathrm{SiO}_{2}$ surfaces. On a thermally grown $\mathrm{SiO}_{2}$ surface, the diffusion coefficients of $\left(\mathrm{H}_{2} \mathrm{O}\right)_{n}^{+}$and $\mathrm{CO}_{3}^{-}$ ions were $2.2 \times 10^{-11} \mathrm{~cm}^{2} / \mathrm{s}$ and $4.8 \times 10^{-12} \mathrm{~cm}^{2} / \mathrm{s}$, respectively. On a chemically cleaned $\mathrm{SiO}_{2}$ surface, the diffusion coefficients of $\left(\mathrm{H}_{2} \mathrm{O}\right)_{n}^{+}$and $\mathrm{CO}_{3}^{-}$ions were $7.5 \times 10^{-9} \mathrm{~cm}^{2} / \mathrm{s}$ and $2.4 \times 10^{-9} \mathrm{~cm}^{2} / \mathrm{s}$, respectively.

The exact solution yields an addition parameter - dielectric thickness, CET. The CET calculation depends only on the initial corona dose, while diffusion coefficient calculation depends only on the radius of corona deposition area.

The present findings are of importance for fundamental understanding of the corona-Kelvin measurements on small test sites below $100 \mu \mathrm{m}$ that are used in silicon IC manufacturing. The method can be expanded to dielectric surfaces other than $\mathrm{SiO}_{2}$.

\section{Acknowledgments}

The authors wish to thank M. Wilson and J. Lagowski for the critical review of the manuscript.

\section{Appendix A. Solution to 2D diffusion with specific boundary conditions}

\section{A.1. Initial conditions}

Corona ions are deposited over a circle of radius $R=$ $40 \mu \mathrm{m}$. Ion density is uniform and is equal to $N_{0}$. Corresponding surface charge density is $Q_{0}=q \times N_{0}$. The wafer diameter is $300 \mathrm{~mm}$, i.e. much larger than the diameter of the corona ion deposition area. 
$2 \mathrm{D}$ diffusion equation is

$$
\frac{\partial N}{\partial t}=D \nabla^{2} N(r, t)=D\left(\frac{\partial^{2} N}{\partial r^{2}}+\frac{1}{r} \frac{\partial N}{\partial r}\right) .
$$

Relation between ion density and surface potential is

$$
q N(r, t)=C_{\text {diel }} \Delta V_{\mathrm{CPD}}(r, t) .
$$

\section{A.2. Solution}

Solution to the diffusion Eq. (A1) satisfying the initial conditions is given by USFKAD [19]:

$$
N(r, t)=N_{0} \int_{0}^{\infty} A(k) J_{0}(k r) \mathrm{e}^{-k^{2} D t} \mathrm{~d} k,
$$

where

$$
A(k)=\int_{0}^{R} J_{0}(k r) k r \mathrm{~d} r=R J_{1}(k R),
$$

where $J$ and $J_{1}$ are the Bessel functions of the first kind of orders zero and one, respectively.

At $r=0$, i.e. in the center of corona ion deposition, the charge density simplifies to

$$
\begin{gathered}
N(0, t)=N_{0} \int_{0}^{\infty} R J_{1}(k R) \mathrm{e}^{-k^{2} D t} \mathrm{~d} k \\
=N_{0} \int_{0}^{\infty} J_{1}(z) \mathrm{e}^{-z^{2} D t / R^{2}} \mathrm{~d} z .
\end{gathered}
$$

Integration by parts produces an equivalent expression

$$
N(0, t)=N_{0}-\frac{4 D^{2} t^{2} N_{0}}{R^{4}} \int_{0}^{\infty} z^{2} J_{1}(z) \mathrm{e}^{-z^{2} D t / R^{2}} \mathrm{~d} z .
$$

Examination reveals that the integral in Eq. (A5) can be reproduced in Eq. (A4) by differentiating with respect to time, so that in fact $N(r, t)$ satisfies the solvable ordinary differential equation

$$
\begin{aligned}
& \frac{\mathrm{d} N(0, t)}{\mathrm{d} t}=\frac{R^{2}}{4 D t^{2}} N(0, t)-\frac{N_{0} R^{2}}{4 D t^{2}} \\
& \quad \Rightarrow N(0, t)=N_{0}-\left[N_{0}-N_{\infty}\right] \mathrm{e}^{-R^{2} / 4 D t} .
\end{aligned}
$$

The value $N_{\infty}=N(0, \infty)$ would be zero if the finite charge was diffused over an idealized infinite region, but we retain it here to enable curve-fitting.

Combining Eqs. (A2) and (A6) gives

$$
\Delta V(t)=\frac{h}{\varepsilon \varepsilon_{0}} q N_{0}-\frac{h}{\varepsilon \varepsilon_{0}} q\left(N_{0}-N_{\infty}\right) \mathrm{e}^{-R^{2} / 4 D t} .
$$

The derivative of Eq. (A7) is $\dot{V}(t)=\frac{\mathrm{d} V}{\mathrm{~d} t}=$ $-\frac{h q\left(N_{0}-N_{\infty}\right) R^{2}}{4 \varepsilon D t^{2}} \mathrm{e}^{-R^{2} / 4 D t}$ and a little algebra produces the remarkable equation

$$
\begin{aligned}
& t \ln \left(-t^{2} \dot{V}(t)\right)=\left(\ln \frac{q\left(N_{0}-N_{\infty}\right) R^{2} h}{4 \varepsilon \varepsilon_{0} D}\right) t-\frac{R^{2}}{4 D} \\
& \quad=\text { slope } \cdot t+\text { intercept. }
\end{aligned}
$$

Thus the plot of $t \times \ln \left(-t^{2} \dot{V}(t)\right)$, defined as a Snider plot, should be a straight line, whose slope and intercept can be estimated by linear regression, enabling the extraction of $D$ and $h$ :

$$
D=\frac{-R^{2}}{4 \times \text { intercept }}, \quad N_{0}-N_{\infty}=\frac{1}{q} \frac{\varepsilon \varepsilon_{0}}{h} \frac{4 D}{R^{2}} \mathrm{e}^{\text {slope }}
$$

As a result Eqs. (A7) and (A9) imply

$$
h=\left(V(t)+\frac{h}{\varepsilon \varepsilon_{0}} q\left(N_{0}-N_{\infty}\right) \mathrm{e}^{-R^{2} / 4 D t}\right) \frac{\varepsilon \varepsilon_{0}}{q N_{0}}
$$

$$
=\left(V(t)+\frac{4 D}{R^{2}} \mathrm{e}^{\text {slope }} \mathrm{e}^{\frac{\text { intercept }}{t}}\right) \frac{\varepsilon \varepsilon_{0}}{q N_{0}},
$$

enabling the calculation of $h$ from the data. When $\varepsilon=\varepsilon\left(\mathrm{SiO}_{2}\right)$ the calculated thickness corresponds to capacitance equivalent thickness, CET.

\section{References}

[1] D.K. Schroder, Meas. Sci. Technol. 12, R16 (2001).

[2] D.K. Schroder, Mater. Sci. Eng. B 91-92, 196 (2002).

[3] P. Edelman, A. Savtchouk, M. Wilson, J. D'Amico, J.N. Kochey, D. Marinskiy, J. Lagowski, Europ. Phys. J. Appl. Phys. 27, 495 (2004).

[4] M. Wilson, D. Marinskiy, A. Byelyayev, J. D'Amico, A. Findlay, L. Jastrzebski, J. Lagowski, ECS Trans. 3, 3 (2006).

[5] R. Williams, M.H. Woods, J. Appl. Phys. 44, 1026 (1973).

[6] R.B. Comizzoli, J. Electrochem. Soc. 134, 424 (1987).

[7] P. Edelman, D. Marinskiy, C. Almeida, J.N. Kochey, A. Byelyayev, M. Wilson, A. Savtchouk, J. D'Amico, A. Findlay, L. Jastrzebski, J. Lagowski, Mater. Sci. Semicond. Proc. 9, 252 (2006).

[8] M.L. Green, M.-Y. Ho, B. Busch, G.D. Wilk, T. Sorsch, T. Conard, B. Brijs, W. Vandervorst, P.I. Räisänen, D. Muller, M. Bude, J. Grazul, J. Appl. Phys. 92, 7168 (2002).

[9] W. Tsai, R.J. Carter, H. Nohira, M. Caymax, T. Conard, V. Cosnier, S. DeGendt, M. Heyns, J. Petry, O. Richard, W. Vandervorst, E. Young, C. Zhao, J. Maes, M. Tuominen, W.H. Schulte, E. Garfunkel, T. Gustafsson, Microelectron. Eng. 65, 259 (2003).

[10] M.M. Shahin, J. Chem. Phys. 45, 2600 (1966).

[11] M.M. Shahin, Appl. Opt. 8, 106 (1969).

[12] D.K. Schroder, Semiconductor Material and Device Characterization, 2nd ed., Wiley, New York 1998, p. 481.

[13] J.A. Voorthuyzen, K. Keskin, P. Bergveld, Surf. Sci. 187, 201 (1987).

[14] A.D. Martin, K.J. McLean, J. Appl. Phys. 48, 2950 (1977).

[15] Y. Awakuni, J.H. Calderwood, J. Phys. D, Appl. Phys. 5, 1038 (1972).

[16] J. D'Amico, Ph.D. thesis, University of South Florida, Tampa 2000.

[17] http://www.semilab.hu/products/si/ faast-300-sl

[18] S.M. Sze, Physics of Semiconductor Devices, Wiley, New York 1981, Ch. 9.

[19] S. Kadamani, A.D. Snider, "USFKAD: An Expert System for Partial Differential Equations", Comp. Phys. Commun. 176, 62 (2007).

[20] S.D. Hossain, M.F. Pas, J. Electrochem. Soc. 140, 3604 (1993).

[21] K.T. Lee, S. Raghavan, Electrochem. Solid-State Lett. 2, 172 (1999). 